\title{
Visual Feedback to Reduce the Negative Effects of Message Transfer Delay on Voice Chatting
}

\author{
Kazuyoshi Murata ${ }^{1}$, Megumi Nakamura $^{2}$ Yu Shibuya ${ }^{2}$, Itaru Kuramoto ${ }^{2}$, \\ and Yoshihiro Tsujino ${ }^{2}$ \\ ${ }^{1}$ Tokyo University of Agriculture and Technology, 2-24-16 Koganei-shi Nakacho, \\ Tokyo. 1848588, Japan \\ ${ }^{2}$ Kyoto Institute of Technology, Matsugasaki Goshokaidocho, Sakyo-ku, \\ Kyoto, 6068588, Japan \\ kmurata@cc.tuat.ac.jp, \{megumi, kuramoto\}@hit.is.kit.ac.jp, \\ \{shibuya, tsujino\}@kit.ac.jp
}

\begin{abstract}
Voice chat system is one of the most popular communication systems using computer networks. There is considerable delay to transfer the sender's voice to the receiver on usual voice chat system. The message transfer delay often causes unintentional interruptions and makes participants of the system feel confused. It is considered that such negative effects occur because participants can't know the status of their voice playback in remote site. In this paper, two types of remote status monitoring systems are introduced to reduce unintentional interruptions and confused feeling. Then, it is confirmed experimentally that these systems were effective to reduce these problems.
\end{abstract}

Keywords: Voice Chatting, Message Transfer Delay, Unintentional Interruption, Status Monitor.

\section{Introduction}

Voice chat system is one of the most popular systems for real-time communication using computer network. However, voice chat system still involves some problems to be solved. For example, message transfer delay is a problem one should not ignore. Message transfer delay on voice chatting often causes unintentional interruptions. These interruptions disrupt flow of the conversation and this disruption makes participants of voice chatting feel confused. It is considered that such problems occur because participants of voice chatting can't know the status of their voice playback in remote site. In this paper, two types of remote status monitoring systems are introduced to reduce the problem caused by delay, and, then, the effectiveness of these systems is confirmed experimentally.

There are many studies about the influence of delay on media communication. For example, Krauss and Bricker[1] analyzed effect of message transfer delay for audiocommunication and they showed that delay caused disruptive influence on communication. Ruhleder and Jordan[2] focused on the impact of delay on distributed communication and they showed that delay disrupts the turn-taking system. Florian and Reichl [3] addressed influences of delay on communication interactivity using 
VoIP connection. Bouch et al.[4] focused on user's perceptions of QoS and presented minimum threshold of acceptable delay in various communication tools. There are many studies aimed to reduce transmission delay. In voice chat system, however, it is impossible to get rid of delay completely. The purpose of our research is not decreasing the length of delay, but reducing the influence of delay by improving user interface of voice chat system.

\section{Impact of Delay on Voice Chat}

Unintentional interruption is one of the most important problems caused by message transfer delay in voice chatting. Fig.1 illustrates an example of conversation in delayed situation. $\mathrm{A} 1, \mathrm{~A} 2$, and $\mathrm{B} 1$ mean that participant $\mathrm{A}$ or participant $\mathrm{B}$ utters something. A1', A2' and B1' mean that participant A or participant B is hearing the utterance of the other participant. If there is not message transfer delay, the voice of participant A is transmitted to participant B's site right away and participant B can receive the voice immediately. If there is certain delay, however, each participant receives delayed utterance of other participant. Therefore the start timing and end timing of A1, A2, and B1 don't coincide with that of A1', A2', and B1'. In this example, participant A utters something first (A1), and waits participant B's response but there is no response. After a certain time, participant A starts talking about another topic (A2) because he/she assumes that participant B doesn't want to respond. Then participant A is interrupted by utterance of participant B (B1') suddenly and he/she is confused. On the other hand, in participant B's site, participant B receives delayed utterance (A1'). Participant B starts responding to participant A after a certain thinking time (B1), but he/she is interrupted by next utterance of A (A2') suddenly. Participant B is also confused because the topic of A2 is not related to A1' and B1.

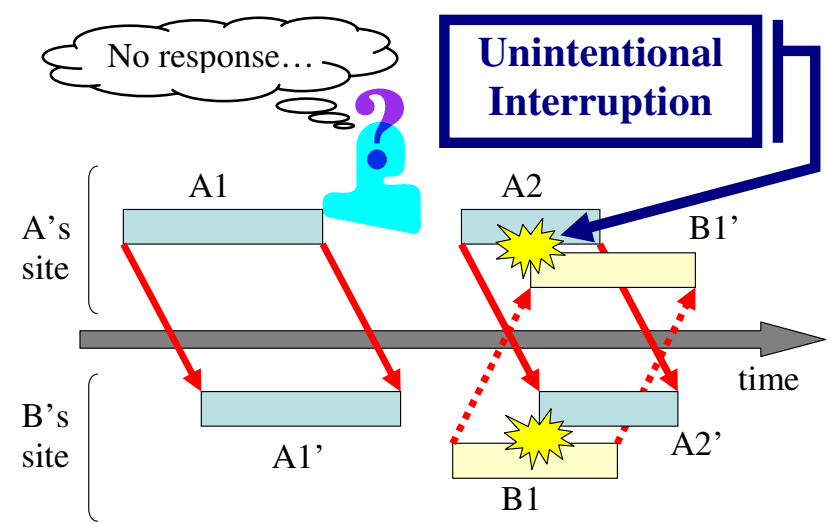

Fig. 1. Conversation in delayed situation

Thus, when there is certain delay in voice chatting, the sequence of conversation is shuffled and unintentional interruptions happen in both of A's site and B's site. These unintentional interruptions disrupt conversation and makes participants confused. Unintentional interruptions also happen even if there is little delay. However, these 
interruptions don't cause disruption of conversation because participants can recover from the disrupted situation easily. In contrast, if the participant utters something to recover in delayed situation, this utterance causes another interruption and this interruption makes the situation worse.

The reason for these problems is that participant A can't wait participant B's response on the assumption that participant B isn't willing to respond. Therefore, if participant A can recognize the length of waiting time caused by delay, it becomes easy for him/her to wait for participant B's response and unintentional interruptions will decrease.

\section{Status Monitoring System}

In this paper, Literal Indicator and Progress Bar Indicator are presented as the status monitoring systems for reducing the negative effects of message transfer delay on voice chatting. Each system has a simple message display window which displays the status message of voice playback in remote site. Users of these systems can understand that his/her partner of conversation is hearing his/her voice or not because he/she can recognize the start timing and end timing of voice playback in remote site easily. Fig.2 illustrates an example of display timing of the status message of voice playback in remote site. The display timing of the status message is delayed for the length of message transfer delay from actual start timing of the voice playback in remote site. That is, the length of the partner's thinking time in remote site $\left(T_{R}\right)$ is equal to the length of it in local site $\left(T_{L}\right)$. Consequently, the participant can distinguish between waiting time caused by message transfer delay and his/her partner's thinking time.

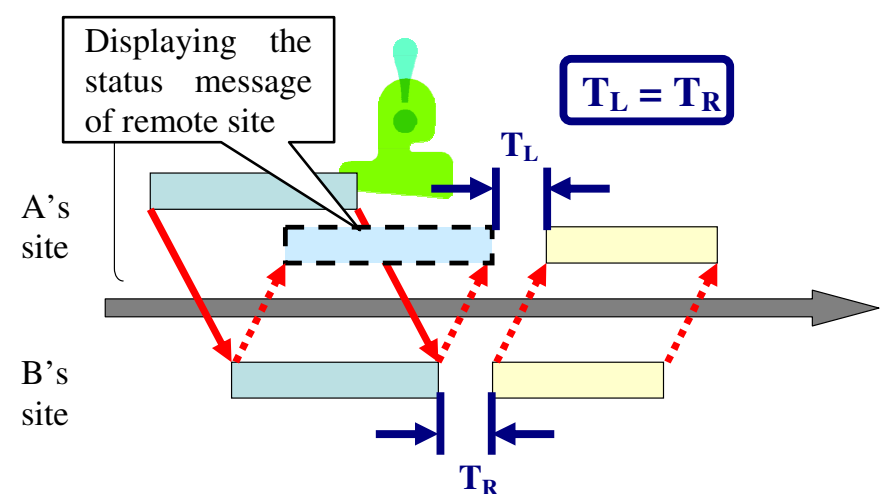

Fig. 2. Display timing of the status message of voice playback in remote site

There are some push-to-talk systems for voice chatting and these systems can prevent unintentional interruptions. However, these systems also prevent "intentional interruptions" in the same way. Intentional interruption is important for turn-taking system. Therefore, it should be avoided for smooth voice chatting to prevent the intentional interruption. Participants using our status monitoring systems can use intentional interruptions, if necessary. 


\subsection{Literal Indicator}

Literal Indicator is illustrated in Fig.3(a). While a participant's voice is playing in remote site, Literal Indicator displays a message which indicates that his/her voice is playing in remote site. When the remote chat client starts playing the participant's voice, a start signal is sent from the remote chat client to the Literal Indicator. Then, Literal Indicator starts to display message when the signal reaches to it. In the same way, when the remote client stops playing participant's voice, a stop signal is sent from the remote client and Literal Indicator stops displaying the message. The participant using Literal Indicator can know if his/her partner in remote site is listening his/her voice or not.

\subsection{Progress Bar Indicator}

Progress Bar Indicator is illustrated in Fig.3(b). Progress Bar Indicator displays a white bar and a red bar, which indicates a process of playing participant's voice in local and remote site. When a participant using Progress Bar Indicator starts to talk, Progress Bar Indicator displays a white bar firstly. The white bar keeps extending while he/she speaks, which means how long the participant is speaking. Then, Progress Bar Indicator displays a red bar over the white bar when Progress Bar Indicator receives a start signal from remote site. The red bar keeps extending until Progress Bar Indicator receives a stop signal from remote site, which means how long the other participant is listening in remote site. The participant using Progress Bar Indicator can know the start timing and end timing of his/her voice playback and infer how long his/her voice will be played in remote site.

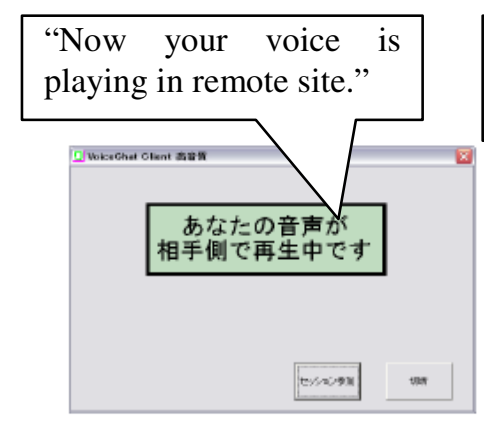

(a)

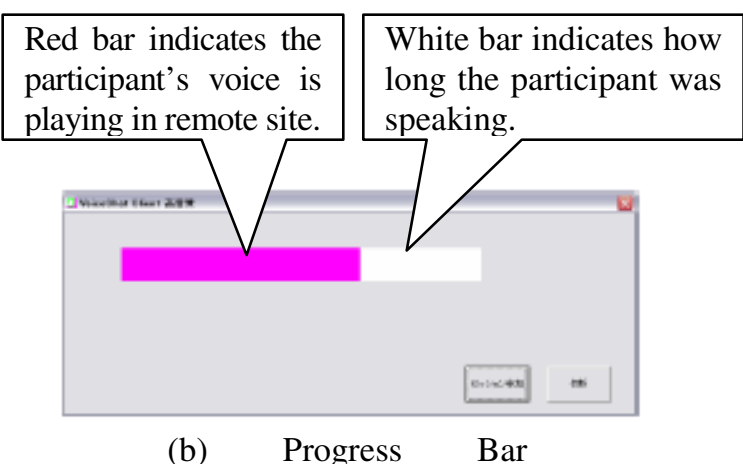

(b) Progress Bar

Fig. 3. Two types of status monitoring systems

\section{Experiment}

An experiment had been performed to confirm that presented status monitoring systems can decrease unintentional interruptions and reduce confused feeling of participants caused by these interruptions. 


\subsection{Experimental Setup}

A simple server-client voice chat system was constructed for this experiment. This chat system had one chat server and two chat client. Each client was connected to the server through LAN and the server could generate some kinds of message transfer delay. This experiment was performed under three kinds of delay conditions with delay of $0.5[\mathrm{sec}], 1.0[\mathrm{sec}]$, and $1.5[\mathrm{sec}]$. There were 10 pairs of subjects who were recruited from our laboratory for this experiment. They had been known well each other and made daily face-to-face communication. Each subject of a pair was located to a soundproofing room and asked to make 10 minutes discussion with another subject. They had a list and there were 20 items in the list. Their task was to rank these items by their mutual consent. For example, there were 20 countries in the list and subjects were asked to discuss which country was good for next journey and rank the countries. Subjects were asked to perform this task by using Literal Indicator and Progress Bar Indicator. Moreover, they were asked to perform this task without these status monitoring systems as the controlled condition. Consequently, there were three kinds of delay conditions $(0.5[\mathrm{sec}], 1.0[\mathrm{sec}], 1.5[\mathrm{sec}])$ and three kinds of status monitoring conditions (Literal Indicator, Progress Bar Indicator, Without status monitor).

In this experiment, the rate of unintentional interruptions was measured. For subjective evaluation, each subject was asked to complete a questionnaire which had a list of following questions after each discussion.

- Q1: You feel confusion about conversation in the discussion. (1: disagree $\sim 5$ : agree)

- Q2: You couldn't utter when you wanted to. (1: disagree 5: agree)

- Q3: Did you change conversational matter when you used the status monitoring system? (Free format)

\subsection{Result and Discussion}

The average rate of unintentional interruptions is illustrated in Fig.4. There was a significant main effect of status monitoring condition $[\mathrm{F}(2,72)=15.272, \mathrm{p}<.01]$, and the rate of without status monitor condition was significantly higher than the rate of Literal Indicator and Progress Bar Indicator condition (Scheffe's test, $\mathrm{p}<.01$ ). This result indicates that status monitoring systems could decrease unintentional interruptions as we expected. From the answers of Q3 ("Did you change conversational matter when you used the status monitoring system?"), many subjects addressed that they waited their partner's response until the displayed message disappeared. It is considered that this behavior resulted in decreasing unintentional interruptions.

Fig.5 illustrates the average score of Q1 ("You feel confusion about conversation in the discussion."). There were significant main effects of delay condition $[F(2,72)=7.097, p<.01]$ and status monitoring condition $[F(2,72)=4.683, p<.05]$. This result indicates that subjects felt strong confusion about conversation when the length of delay was 1.5 [sec] but the status monitoring systems could reduce the confused feeling of participants. Especially, the score with Literal Indicator was significantly lower than the score without status monitor [Scheffe's test, $\mathrm{p}<.05]$. 


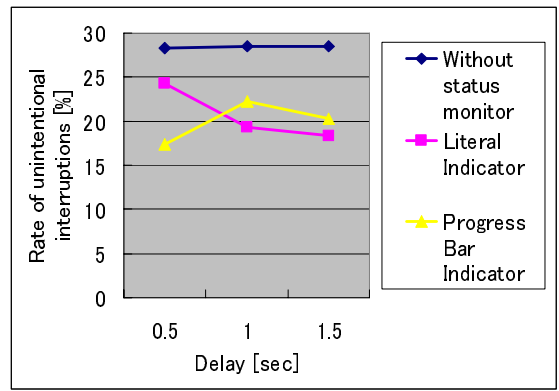

Fig. 4. The average rate of unintentional interruptions

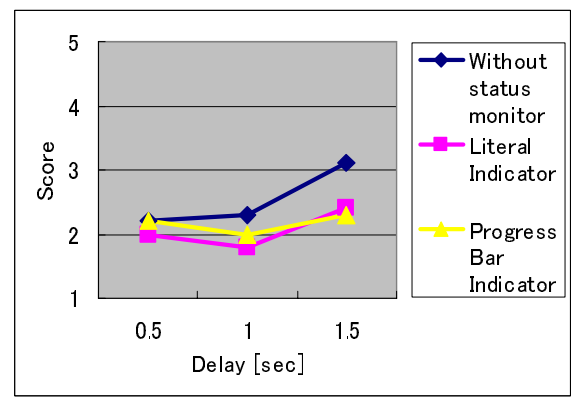

Fig. 5. The average score of Q1: You feel confusion about conversation in the discussion (1: disagree $~ 5:$ agree)

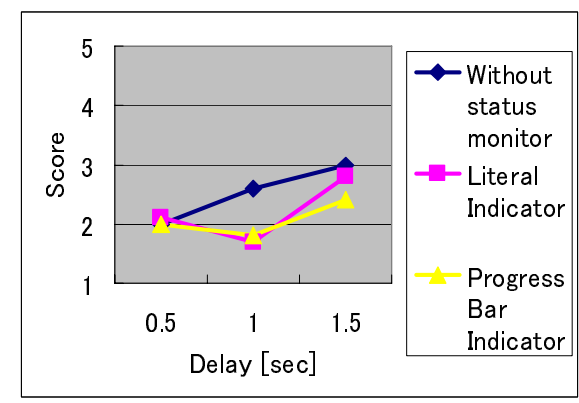

Fig. 6. The average score of Q2: You couldn't utter when you wanted to. (1: disagree $\sim 5$ : agree)

Fig.6 illustrates the average score of Q2 ("You couldn't utter when you wanted to."). There were also significant main effects o f delay condition $[F(2,72)=13.769$, $\mathrm{p}<.01]$ and status monitoring condition $[\mathrm{F}(2,72)=4.871, \mathrm{p}<.05]$, and the score with Progress Bar Indicator was significantly lower than the score without status monitor [Scheffe's test, $\mathrm{p}<.05]$. It is important for voice chatting that participants can utter when they want. In this experiment, subjects without status monitoring system felt difficult to utter as the delay was increased. However, if they could use status monitoring systems, especially Progress Bar Indicator, they could utter when they wanted.

We considered that unintentional interruptions would increase with increasing delay before this experiment. However, the result showed that these interruptions didn't increase actually. On the other hand, the results of questionnaire showed that confusion of subjects increased with increasing delay as we expected. From these results, it is considered that the unintentional interruptions in long delay condition can confuse subjects strongly. Furthermore, it is more important to decrease these unintentional interruptions in long delay condition than in short delay condition. Literal Indicator and Progress Bar Indicator were effective to decrease unintentional interruptions in both of short delay condition and long delay condition, and, 
moreover, these could be effective for reducing the confusion about conversation in long delay condition.

It was not clear the difference between two types of status monitoring systems from the result of this experiment. However, many subjects indicate that it was easier for the Progress Bar Indicator to understand the process of their voice playback in remote site and to predict the timing of the response. This difference will be clarified by further experiment and investigation in the future.

\section{Conclusion and Future Work}

In this paper, we focused on unintentional interruptions caused by message transfer delay in voice chatting and confused feeling caused by the unintentional interruptions. To reduce the negative effects, we presented two types of status monitoring systems, Literal Indicator and Progress Bar Indicator. Then we had an experiment to confirm the effect of these systems. From the result of this experiment, we confirmed that these systems were effective to decrease unintentional interruptions and could reduce the confusion about conversation even if there were certain amount of delay. However, further investigation is needed for improving presented systems. For example, it is important for improvement of these systems to compare Literal Indicator and Progress Bar Indicator in detail. In addition, it is needed to evaluate other display styles instead of text or progress bar. Future work focuses on improving these systems and building them into practical applications.

\section{References}

1. Krauss, R., Bricker, P.: Effect of Transmission Delay and Access Delay on the Efficiency of Verbal Communication. The Journal of the Acoustical Society of America 41(2), 286-292 (1967)

2. Ruhleder, K., Jordan, B.: Co-Constructing Non-Mutual Realities: Delay-Generated Trouble in Distributed Interaction. Computer Supported Cooperative Work. 10(1), 113-118 (2001)

3. Florian, H., Reichl, P.: The Well-Tempered Conversation: Interactivity, Delay and Perceptual VoIP Quality. In: Proc. IEEE ICC'05, pp. 244-249 (2005)

4. Bouch, A., Sasse, M., DeMeer, H.: Of Packets and People: A User-centered Approach to Quality of Service. In: Proc. IWQoS'00, pp. 189-197 (2000) 